\title{
SELECTED ABSTRACTS
}

Rohan Siriwardena

\section{Surgical management of disappearing colorectal liver metastases}

British Journal of Surgery. 2013 Oct;100(11):1414-20

Bischof DA, Clary BM, Maithel SK, Pawlik TM.

\section{Background}

Owing to expanded surgical indications for colorectal liver metastasis (CRLM) and improved systemic therapy, hepatic surgeons are increasingly faced with the problem of disappearing (no longer visible on imaging) liver metastasis (DLM).

\section{Methods}

A review of relevant studies was performed. Studies that reported on DLM associated with preoperative chemotherapy for CRLM were identified, and data were synthesized and tabulated. The PubMed database was searched for relevant articles published between January 2000 and December 2012.

\section{Results}

A complete response on imaging does not necessarily equate with a complete clinical or pathological response. Rather, residual macroscopic disease is found in about 25-45 per cent of patients at the time of operation. Even among patients with a complete pathological response, long-term remission occurs in only 20-50 per cent of those treated with systemic therapy. A durable response of DLM is more common following the use of hepatic artery infusion therapy.

\section{Conclusion}

Liver resection should include all original sites of disease if possible

\section{Commentary:}

Arinda Dharmapala

Hon Consultant Surgeon, Teaching Hospital Peradeniya \& Senior lecturer, Dept of Surgery, Faculty of Medicine, University of Peradeniya

The review shows no clear consensus on the guidelines to manage disappearing colorectal liver metastasis following preoperative chemotherapy. Over the years, preoperative chemotherapy regimens have improved and tumour response rates from $14 \%$ to $58 \%$ till 2008. Conversion chemotherapy regimes show a $50 \%$ response rate, enabling surgeons to do curative resections in $20 \%$ of patients. According to many studies, after chemotherapy 5-20\% of CRLM disappears completely in cross sectional imaging. The percentage of DLM difference could be due to the different imaging modality used in each study as the MRI is more sensitive in detecting lesions as opposed to CT scans. Secondly this may be related to the type of chemotherapy used i.e. hepatic arterial infusion therapy (HAI) that has proved better over systemic chemotherapy. The steatosis and steatohepatitis which occur following chemotherapy reduces the sensitivity and specificity of CT scanning and metastases are missed in imaging. The effect of this on MRI is minimal. Complete imaging response did not denote complete pathological disappearance. It is only seen in $15-70 \%$ of patients. Therefore it is necessary for the surgeons to consider minimum metatasectomy or paranchymal sparing resections as disease free survival significantly improves with resection even though the overall survival shows no benefit.

CRLM should be considered a metachronous disease as studies show that the recurrence of CRLM is high after primary liver resection. Subsequent resection gives a better prognosis than chemotherapy. Thus surgeons must consider paranchymal sparing resections when dealing with DLMs.

Surgeon and breast unit volume-outcome relationships in breast cancer surgery and treatment

Annals of Surgery 2013 Nov;258(5):808-14

McDermott AM, Wall DM, Waters PS, Cheung S, Sibbering M, Horgan K, Kearins O, Lawrence G, Patnick J, Kerin MJ.

\section{Objectives}

To determine whether surgeon case volume and Unit 
case volume affected specific recognized key performance indicators (KPIs) of breast cancer surgical management.

\section{Background}

An increasing body of evidence suggests that a higher standard of cancer care, demonstrated by improved outcomes, is provided in high-volume units or by high-volume surgeons. The volume-outcome relationship pertaining to screen-detected breast cancers has yet to be thoroughly established and remains a pertinent issue in view of the debate surrounding breast cancer screening.

\section{Methods}

The study population comprised all women with a new screen diagnosed breast cancer between 20042005 and 2009-2010. Surgeons' mean annual patient volumes were calculated and grouped as very low $(<5)$, low (5-15), medium (16-49), or high volume $(>50)$. The effect of breast screening unit volume was also evaluated. Statistical analyses were performed using Minitab V16.0 software (State College, PA) and R V2.13.0.

\section{Results}

There were 81,416 patients aged $61( \pm 6.8)$ years treated by 682 surgeons across 82 units. There were 209 very low-, 126 low-, 295 medium-, and 51 highvolume surgeons. The proportion of patients managed by very low-, low-, medium-, and high-volume surgeons was $1.2 \%, 6.9 \%, 65.5 \%$, and $25.7 \%$, respectively. Patients managed by high-volume surgeons were more likely to have breast-conserving surgery (BCS) than those managed by low-volume surgeons $(\mathrm{P}<0.001)$. There was a higher proportion of sentinel lymph node biopsies (SLNB) performed by high-volume surgeons in invasive cancers $(\mathrm{P}=0.005)$. High-volume units performed more BCS and SLNB than low-volume units $(\mathrm{P}<0.001$ and $\mathrm{P}<0.001$, respectively).

\section{Conclusions}

Even in a setting with established quality control measures (KPIs) surgeon and unit volume have potent influences on initial patient management and treatment

\section{Commentary:}

Samira Janaka Jayasinghe

Consultant Surgeon, Military Hospital

The UK currently offers mammography every 3 years to all women aged between 50 and 70 in order to screen for breast cancer. These screen detected patients receive a variety of treatment modalities such as, wire guided excision, breast conservation surgery and sentinel lymph node biopsy. Traditionally, breast cancer patients underwent more radical procedures like mastectomy and axillary clearance which have a higher chance of debilitating complications. Presently, these more radical procedures are limited to those who are not amenable to breast conservation surgery.

Ailbhe et al had studied how the surgeon case volume and unit case volume affect the patient management outcome in breast cancer. The study had shown that surgeons and units with high case volume (case load) are likely to perform more breast conservation surgery and sentinel lymph node biopsy, which are less radical procedures.

However, we in Sri Lanka, do not have specialised breast units so far. Hence, establishing specialised breast units may improve the patient management outcome in the combat against the commonest cancer among Sri Lankan females.

\section{Bariatric surgery and prevention of type 2 diabetes in Swedish obese subjects}

New England journal of medicine. 2012 Aug 23;367(8):695-704

Carlsson LM, Peltonen M, Ahlin S, Anveden A, Bouchard C, Carlsson B, Jacobson P, Lönroth H, Maglio C, Näslund I, Pirazzi C, Romeo S, Sjöholm K, Sjöström E, Wedel H, Svensson PA, Sjöström L.

\section{Background}

Weight loss protects against type 2 diabetes but is hard to maintain with behavioral modification alone. In an analysis of data from a nonrandomized, prospective, controlled study, we examined the effects of bariatric surgery on the prevention of type 2 diabetes.

\section{Methods}


In this analysis, we included 1658 patients who underwent bariatric surgery and 1771 obese matched controls (with matching performed on a group, rather than individual, level). None of the participants had diabetes at baseline. Patients in the bariatric-surgery cohort underwent banding (19\%), vertical banded gastroplasty $(69 \%)$, or gastric bypass $(12 \%)$; nonrandomized, matched, prospective controls received usual care. Participants were 37 to 60 years of age, and the body-mass index (BMI; the weight in kilograms divided by the square of the height in meters) was 34 or more in men and 38 or more in women. This analysis focused on the rate of incident type 2 diabetes, which was a prespecified secondary end point in the main study. At the time of this analysis (January 1, 2012), participants had been followed for up to 15 years. Despite matching, some baseline characteristics differed significantly between the groups; the baseline body weight was higher and risk factors were more pronounced in the bariatric-surgery group than in the control group. At 15 years, $36.2 \%$ of the original participants had dropped out of the study, and $30.9 \%$ had not yet reached the time for their 15year follow-up examination.

\section{Results}

During the follow-up period, type 2 diabetes developed in 392 participants in the control group and in 110 in the bariatric-surgery group, corresponding to incidence rates of 28.4 cases per 1000 person-years and 6.8 cases per 1000 person-years, respectively (adjusted hazard ratio with bariatric surgery, 0.17; $95 \%$ confidence interval, 0.13 to $0.21 ; \mathrm{P}<0.001)$. The effect of bariatric surgery was influenced by the presence or absence of impaired fasting glucose $(\mathrm{P}=0.002$ for the interaction) but not by $\mathrm{BMI}(\mathrm{P}=0.54)$. Sensitivity analyses, including end-point imputations, did not change the overall conclusions. The postoperative mortality was $0.2 \%$, and $2.8 \%$ of patients who underwent bariatric surgery required reoperation within 90 days owing to complications.

\section{Conclusions}

Bariatric surgery appears to be markedly more efficient than usual care in the prevention of type 2 diabetes in obese persons. (Funded by the Swedish Research Council and others; Clinical Trials.gov number, NCT01479452.)

\section{Commentary:}

Thejana Wijeratne

Hon Consultant Surgeon \& Senior Lecturer, CSTH

The Swedish obesity study is an ongoing government funded research which commenced in 1987 with more than 4000 patients who underwent bariatric surgery in Sweden. Several landmark papers which were instrumental in setting standards in the field of bariatric surgery have emerged from this study group. The above study compare the risk of development of Diabetes in a prospective manner in a post bariatric surgery group compared to a control group over a 15 year follow up period. Association between diabetes and morbid obesity is well proven and remission of diabetes after successful bariatric surgery is also well documented. This study highlights the role of bariatric surgery as a preventive measure in future occurrence of diabetes in a high risk group compared to a conservatively managed control group. Despite a less favourable risk profile at baseline in development of future diabetes; the post bariatric surgery group showed a statistically significant risk reduction in development of diabetes over 15 year follow $\mathrm{up}(\mathrm{P}<0.001)$. As diabetes is a disease with many risk factors, the hazard ratios were calculated in both groups after multi-variable adjustments. The validity of this unique study was maintained with large sample sizes in both study and control groups (adequately powered) and with continued long term follow up.

Bariatric surgery is still in its infancy in Sri Lanka where obesity and incidence of diabetes is increasing at an alarming rate. The results of the above study clearly proves that bariatric surgery is effective not only as a "curative" option in management of obesity associated diabetes; but also preventing diabetes in the long term.

Timing of colonoscopy after resection for colorectal cancer: are we looking too soon?

Diseases of Colon and Rectum. 2013 Nov;56(11):1233-6.

Cone MM, Beck DE, Hicks TE, Rea JD, Whitlow CB, Vargas HD, Margolin DA. 


\section{Background}

Based on current National Comprehensive Cancer Network guidelines, colonoscopic surveillance after colorectal cancer resection should begin at 1 year.

\section{Objective}

The aim of this study was to determine whether the incidence of cancer or advanced polyp detection rate was high enough to justify colonoscopy at 1 year.

\section{Design}

The Ochsner Clinic Tumor Registry Database was queried for patients who underwent a segmental colectomy or proctectomy between 2002 and 2010 . Patients who had a preoperative colonoscopy and at least 1 documented postoperative colonoscopy were included. We considered new cancer or polyps of $\geq$ $1 \mathrm{~cm}$ as missed on the preoperative colonoscopy. Patients with an identified genetic trait causing a predisposition to colorectal cancer were excluded.

\section{Results}

Five hundred twelve patients underwent resection, and 155 met our inclusion criteria. The average age was 64 years, and 53\% patients were male. There were $32.9 \%$ with stage I disease, $35 \%$ with stage II disease, $27.1 \%$ with stage III disease, and $5.2 \%$ with stage IV disease. Of these patients, $52.2 \%$ had a right colectomy, $7.1 \%$ had a left colectomy, $16.8 \%$ had a sigmoid colectomy, $22 \%$ had a low anterior resection, and $1.3 \%$ had a transanal resection. The average time to first postoperative colonoscopy was 478 days (SD \pm 283 days). Twenty-four patients had adenomatous polyps detected on their first surveillance colonoscopy, but only $5(3.2 \%)$ polyps were $\geq 1 \mathrm{~cm}$, and there was no correlation between stage of cancer and finding a polyp. No new cancers were detected, but $3(1.9 \%)$ had an anastomotic recurrence.

\section{Conclusions}

The performance of surveillance colonoscopy at 1 year resulted in the detection of only 5 missed polyps $\geq 1 \mathrm{~cm}$ and no metachronous cancers. Anastomotic recurrences were rare, and the majority were in patients who had rectal cancer that could be evaluated by in-office flexible sigmoidoscopy. Extending the time to first colonoscopy appears to be safe and would help conserve valuable resources, including physician and facility time, which is imperative in the current health care climate

\section{Commentary:}

Wasantha Wijenayake

Consultant Surgeon, AS / NHSL

Current protocols on surveillance after curative resections for colorectal cancer do not show any cancer related survival benefit in carrying out colonoscopy at the end of one year. Even though with a small study population of 155 patients this paper has reflected the same results by detecting only five polyps (3\%) of significant size whilst rectal anastomotic recurrences were 2 out of $3(2 \%)$ recurrences at one year colonoscopy.

In addition others agree with these findings including the American Society of Clinical Oncology recommending that the initial colonoscopy be carried out at the end of 3 years. Considering the increased detection of colorectal cancers and limitations of trained colonoscopists and resources it may be more appropriate for Sri Lanka to adopt a delaying of the first colonoscopy to 2 years after a colonic cancer resection with 6 monthly flexible sigmoidoscopies for rectal cancer surveillance. The importance of high quality preoperative colonoscopy should be emphasized along with this approach.

\section{Answers for Images in Surgery (page 54)}

1. Intrinsic brain tumor, with evidence of haemorrhage and necrosis, with mass effect. Diagnosis is likely to be an intrinsic malignant brain tumor, glioblastoma multiforme

2. It is a performance scoring system used to assess patients with brain tumors

3. The tumor is causing significant mass effect, which causes raised intracranial pressure with eventual brain herniation, leading to Cushings response(Hypertension and bradycardia leading to brain hypoxia and ischaemia ) leading to death. 